\title{
Food Craving, Seeking, and Consumption Behaviors: Conceptual Phases and Assessment Methods Used in Animal and Human Studies
}

\author{
Young Hee Lee ${ }^{1,2,3}$, Meelim Kim ${ }^{1,2,3}$, Miwoo Lee ${ }^{1,2,3}$, Dongju Shin' ${ }^{1}$, Dong-Soo Ha ${ }^{1}$, Joon Seok Park' ${ }^{1}$ You Bin Kim ${ }^{1,2,3}$, \\ Hyung Jin Choi ${ }^{1,2,3,4, *}$ \\ ${ }^{1}$ Functional Neuroanatomy of Metabolism Regulation Laboratory, Department of Anatomy and Cell Biology, ${ }^{2}$ Department of Biomedical Science, and ${ }^{3}$ BK21Plus \\ Biomedical Science Project Team, Seoul National University College of Medicine, Seoul; ${ }^{4}$ Wide River Institute of Immunology, Seoul National University, Hongcheon, \\ Korea
}

What drives us to eat? It is one of the most fundamental questions in the obesity research field which have been investigated for centuries. Numerous novel in vivo technologies in the neuroscience field allows us to reevaluate the multiple components and phases of food-related behaviors. Focused on the cognitive, executive, behavioral and temporal aspects, food-related behaviors can be distinguished into appetitive phase (food craving $\rightarrow$ food seeking) and consummatory phase (food consumption). Food craving phase is an internal state or stage in which the animal has the motivation to eat the food but there is no actual food specific behaviors or actions. Food seeking phase entails repeated behaviors with a food searching purpose until the animal discovers the food (or food-related cue) and the approach behavior stage after the discovery of food. Food consumption phase is the step that the animal grabs, chews and intake the food. This review will specifically focus on characteristics and evaluation methods for each phase of food-related behavior in rodent, non-human primates and human.

Key words: Hunger, Hypothalamus, Behavior
Received June 19, 2019

Reviewed July 11, 2019

Accepted August 10, 2019

${ }^{*}$ Corresponding author

Hyung Jin Choi

(i)

https://orcid.org/0000-0003-0593-6978

Department of Biomedical Sciences and Department of Anatomy and Cell Biology, Seoul National University College of Medicine, 103 Daehak-ro, Jongno-gu, Seoul 03080, Korea

Tel: +82-2-740-8204

Fax: +82-2-745-9528

E-mail: hjchoi@snu.ac.kr

\section{INTRODUCTION}

Hunger is one of the strongest behavioral drive in the animal kingdom. Traditional neuronal paradigm for feeding behavior is that agouti-related peptide (AgRP) neurons which are located in the hypothalamus are critical for the whole sequence of food-related behavior (food craving $\rightarrow$ food seeking $\rightarrow$ food consumption). ${ }^{1}$ However, the neuronal activity of AgRP neurons was immediately inhibited when a pellet of chow was presented to fasted mice even before the mice approached and started to eat the chow. ${ }^{2,3}$ This landmark studies revealed that AgRP neurons are only responsible for appetitive part of feeding behavior (food craving and seeking), but not the consumption part of feeding behavior.

The traditional assessment method for feeding behavior is measurement of the consumed food amount. However, feeding involves emotion (craving, motivation), cognition (learning, memory, decision making), sensory (visual, taste, olfactory) and motor behaviors in an interdependent manner. ${ }^{4}$ To address this, several behavior tests have been developed to dissect the respective roles of food-related behaviors. Moreover, optical recordings of neuronal activity in freely moving mice have been devised and applied to study the dynamics of hypothalamic neurons during feeding be-

Copyright (C) 2019 Korean Society for the Study of Obesity

(a) This is an Open Access article distributed under the terms of the Creative Commons Attribution Non-Commercial License (http://creativecommons.org/licenses/by-nc/4.0/) which permits unrestricted non-commercial use, distribution, and reproduction in any medium, provided the original work is properly cited. 
havior tests. In non-human primates and human studies, researchers have used functional magnetic resonance imaging (fMRI), computerized tasks and noncomputerized behavioral tasks for investigating food-related behavior and its neuronal regions.

Thus, in this review, we have dissected food-related behaviors and examined the assessment methods of each behavior by three phases; food craving, food seeking, and food consumption. Food craving phase is the brain state with high desire and preoccupation toward food but does not accompany specific foraging or searching action for food. On the other hand, food seeking phase starts and pursues navigating the putative area or device to obtaining food. Food seeking phase is terminated when animal discovers the food

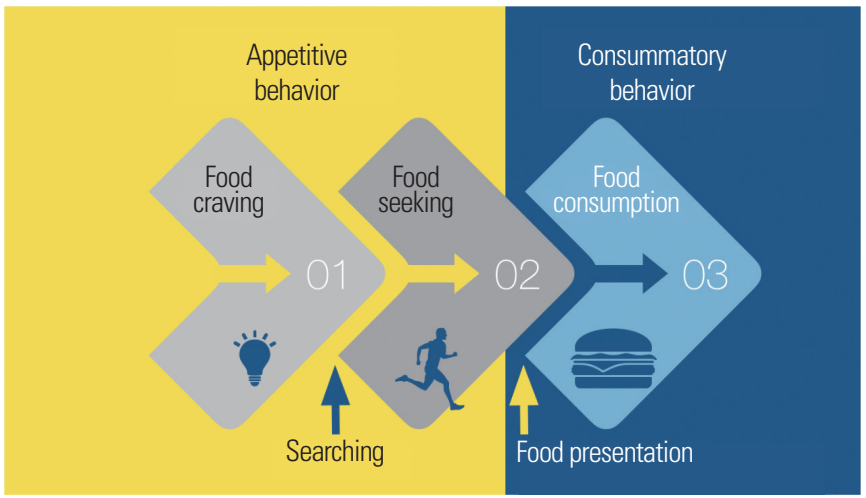

Figure 1. Food craving, seeking, and consumption phases. and approaches it. Food consumption phase begins when animal bites and swallows the food (Fig. 1).

\section{METHODS FOR EVALUATION OF FOOD CRAVING BEHAVIOR}

\section{Rodent}

Food craving is a step in which the organism has the will to eat the food before the identification of food. Thus, mice do not know the existence of the food yet. Because craving is a step before occurrence of actual behavior, it is hard to measure food craving directly via behavior tests (Table 1 ).

\section{Neuronal monitoring}

The craving step is known to be associated with the AgRP neurons and pro-opiomelanocortin neurons in the arcuate nucleus. Sensory detection of food leads to rapid inhibition of AgRP neurons by using optical or electrophysiological recordings. ${ }^{5}$

\section{Behavioral monitoring}

The most commonly known method that is known to be used to understand the motivational drive of mice is the operant chamber test (operant conditioning in Skinner box). ${ }^{6}$ Lever-pressing is a

Table 1. Methods for evaluation of food craving behavior

\begin{tabular}{|c|c|c|c|c|c|}
\hline \multirow{2}{*}{$\begin{array}{l}\text { Feeding phase } \\
\text { Craving }\end{array}$} & \multicolumn{2}{|l|}{ Species } & \multicolumn{2}{|c|}{ Monitoring method } & \multirow{2}{*}{$\begin{array}{l}\text { Author (year) } \\
\text { Chen et al. }(2015)^{2}\end{array}$} \\
\hline & Rodent & Neuronal monitoring & Tool & Optogenetics & \\
\hline & & & & Fiber photometry & Chen et al. $(2015)^{2}$ \\
\hline & & & & Miniscope & Betley et al. $(2015)^{3}$ \\
\hline & & & & Head-fixed optetrode & Mandelblat-Cerf et al. $(2015)^{5}$ \\
\hline & & Behavioral monitoring & Behavior test & Real-time place preference test & Jennings et al. $(2015)^{7}$ \\
\hline & & & & Conditioned place preference test & Tzschentke $(2007)^{8}$ \\
\hline & & & & Home cage behavior analysis & Dietrich et al. $(2015)^{9}$ \\
\hline & & & & Pain response test & Alhadeff et al. $(2018)^{10}$ \\
\hline & Non-human primate & Computerized monitoring & Tool & PET, fMRI & Fukuda and Ono (1993) ${ }^{11}$ \\
\hline & & Behavioral monitoring & Task & Behavior response test & Watanabe et al. $(2001)^{12}$ \\
\hline & Human & Computerized monitoring & Tool & $\mathrm{fMRl}$ & He et al. $(2014)^{13}$ \\
\hline & & & Task & Dot probe task & Johansson et al. $(2004)^{14}$ \\
\hline & & & & Approach-avoidance task & Brockmeyer et al. $(2019,2015)^{15,16}$ \\
\hline & & & Survey & G-FCQ-T/S & Nijs et al. $(2007)^{17}$ \\
\hline & & & & CMO & Sobik et al. $(2005)^{18}$ \\
\hline & & Behavioral monitoring & Behavior test & Attention to cues & Sobik et al. $(2005)^{18}$ \\
\hline
\end{tabular}

PET, positron emission tomography; fMRI, functional magnetic resonance imaging; G-FCQ-T/S, General Food Cravings Questionnaire-Trait/State; CMQ, Craving and Mood Questionnaire. 
conditioned behavior, and the act of pressing the lever has been assumed to be driven by motivation for food without the act of food seeking or consuming. However, such behaviors can be potentially categorized separately as seeking or consumption, and so were recorded in the later parts of the review.

\section{Real-time place preference test}

This assay measures the motivational valence of the rodent in real time. In the test, one side of rectangular chamber is paired with optogenetic stimulation. When the stimulation is rewarding, the rodent spends more time on this side. This method can be used to as an indirect evidence of increased general motivation (including food specific motivation). ${ }^{7}$

\section{Conditioned place preference test}

Motivational valence of the experience can be measured by conditioned place preference test which involves three steps. The first step is habituation which gives the mouse a chance to explore the two sides of the chamber. The second phase is conditioning. In this step, mouse is allowed access only one side of the chamber which is paired with an unconditioned stimulus such as food. Third step is preference testing. In this phase, time spent of the mouse on each side of chamber without unconditioned stimulus is measured. ${ }^{8}$

\section{Home cage behavior analysis}

There is a study that analyzed the developed changes in homecage behaviors (ambulatory activity) when food was not presented to mice. ${ }^{9}$ The cages were located in front of cameras of the Home Cage Scan System (CleverSys, Reston, VA, USA) with infrared radiation light panels. Interestingly, when AgRP neurons are activated, mice showed increase in home cage behavior even though they were sated.

\section{Pain response test}

Time spent licking paw was quantified for behavior response to formalin paw injection. When AgRP neuron were activated, the inflammatory pain response was significantly attenuated. Also food deprived mice showed reduced frequency and duration of the pain response. However, this assay did not affect food consumption. Together, this quantification method can be used to analyze the crav- ing phase specific behavioral test. ${ }^{10}$

\section{Non-human primate}

In human studies, the extent of craving for food can be reported by the subjects through surveys. However, in non-human primates for whom verbal reports of craving are impossible, the assessment of the extent of food-craving is difficult. Some studies have implied that reward processes and their neural mechanisms can be studied about the subjective experience of the subjects without knowledge. ${ }^{19}$ Though, the incapability of subjects to report the self-diagnosed extent of food craving necessitates the use of indirect measures obtained from continual monitoring. Various measures were employed in the literature to quantify the extent of food-craving in non-human primates under different conditions. The assessment methods can be divided into two categories: computerized monitoring and behavioral monitoring.

\section{Computerized monitoring}

The activity of neurons in different parts of the brain is usually recorded when non-human primates are performing behavioral tasks. Single unit activities of the area of interest are measured during operant feeding tasks to clarify the neural circuits involved in the feeding activities. ${ }^{11,20}$ With specific brain regions identified to be in correlation with food-craving, tools including fMRI and positron emission tomography can be more actively employed to assess the motivational state of non-human primate subjects.

\section{Behavioral monitoring}

Besides the use of brain imaging, the observation of subject's behavior is the most frequently used method to assess the motivational state of non-human primates. One study has presented a form of behavioral response test where cynomolgus monkeys ( $\mathrm{Ma}$ caca fascicularis), fixed in primate chair, were trained to press certain buttons upon specific signals. After successful completion of the task, the monkey would be given liquid food as reward, but sometimes the spout would not displace the reward. The rewarded and unrewarded tasks were mixed randomly, maintaining a ratio of 3 to 2. Unrewarded task had to be completed for the monkey to progress to the rewarded task. In this study, the reaction time to expected liquid rewards and the duration of anticipatory licking were re- 
corded. ${ }^{12}$ Such observations at an unrewarded trial could serve as a measure for food craving.

\section{Human}

There are several computerized tasks to assess food craving behaviors for humans. Also, the studies using these computerized tasks with $\mathrm{fMRI}$ indicated that there are some typical brain regions related to the food craving of humans.

\section{Food reward brain regions}

Many fMRI studies investigated activation of reward-system and associated brain regions in response to food image. Previous studies revealed that insula, operculum, amygdala, anterior cingulate cortex, striatum, caudate, putamen and orbitofrontal cortex are significantly activated when encountered with high-calorie food images. ${ }^{21}$

\section{Computerized monitoring}

Dot probe task

Dot probe paradigm assesses attentional bias. ${ }^{14,22}$ Two stimuli, one of which is target stimuli (i.e., food image) and the other is neutral, presented for predetermined length of time (most commonly $500 \mathrm{~ms}$ ). Dot probe is presented in the location of one former stimulus. Subjects are instructed to response the location of dot probe quickly. Attentional bias score is calculated using reaction time for target cue and neutral cue.

\section{Approach-avoidance task}

Subjects push and pull the joy-stick to manipulate food picture stimuli on the screen to move away from or close to them. ${ }^{15}$

\section{General Food Cravings Questionnaire-Trait/State}

General Food Cravings Questionnaire-Trait is composed of nine subscales. Each subscale reflects an aspect of possible aberrant and consequences related to food craving by 39 items. These subscales are (1) motivation to consume food, (2) expectation of positive reinforcement from eating, (3) anticipation of relieving from negative feelings from eating, (4) feasible lack of control over eating, (5) obsession with food, (6) craving accounting for physiological condition, (7) emotions before or during craving or eating, (8) environmental stimulus causing food cravings, and (9) guilt that may result from food cravings. ${ }^{17,23}$ General Food Craving Questionnaire-State is to assess the degree of affirmation with each item at the moment of answering the questionnaire. It consists of five factors: (1) excessive desire to eat, (2) prospect of positive reinforcement from eating, (3) anticipation of relieving from negative feelings from eating, (4) obsession with food or lack of control over eating, and (5) craving along with physiological condition. ${ }^{17,23}$

\section{Craving and Mood Questionnaire}

Craving and Mood Questionnaire (CMQ) is composed of eleven items and were rated on a scale of $0-100$. The items of CMQ were integrated to form a scale evaluating craving for food, adopted from the same items applied to assess craving for cigarettes. ${ }^{18}$

\section{Behavioral monitoring}

One study designed the experimental portion of the session using attention to cues along with five trials: pencil cue (neutral stimulus cue), food cue (the preferred food of each individuals), and three eating trials. The purpose of the pencil cue trial is to assess a baseline measure of craving. In addition, participants were asked to focus on the food cue, imagining about what it would be like to eat during the food cue trial. Then the participants filled out forms which consist of craving, mood, and rating of the food. ${ }^{18}$

\section{METHODS FOR EVALUATION OF FOOD SEEKING BEHAVIOR}

\section{Rodent}

Detailed information regarding feeding circuits have been elucidated by monitoring neuronal activity in awake behaving mice. ${ }^{2}$ Contrary to expectations, several calcium dynamics studies showed lower activity of AgRP neurons when a mouse is actually eating a food pellet compared to the phase before discovering the food pellet. ${ }^{3,5}$ It implies a possibility that AgRP neuron activation during food craving phase transmits a sustained signal to a population of its down-stream neurons. ${ }^{24}$ This sustained signal from AgRP neurons could serve as a sustained drive to continue on consummatory behavior (even after the rapid decline in AgRP neuron activity induced by sensory detection of food) (Table 2).,3 
Table 2. Methods for evaluation of food seeking behavior

\begin{tabular}{|c|c|c|c|c|c|}
\hline \multirow{2}{*}{$\begin{array}{l}\text { Feeding phase } \\
\text { Seeking }\end{array}$} & Species & \multicolumn{3}{|c|}{ Monitoring method } & \multirow{2}{*}{$\begin{array}{c}\text { Author (year) } \\
\text { Shang et al. }(2019)^{25}\end{array}$} \\
\hline & Rodent & Neuronal monitoring & Tool & Optogenetics & \\
\hline & & & & Optogenetics & Jennings et al. (2015) ${ }^{7}$ \\
\hline & & & & Fiber photometry & Shang et al. $(2019)^{25}$ \\
\hline & & Behavioral monitoring & Behavior test & Cricket hunting test & Zhao et al. $(2019)^{26}$ \\
\hline & & & & Operant conditioning chamber & Chen et al. $(2019)^{27}$ \\
\hline & & & & Buried food seeking test & Machado et al. $(2018)^{28}$ \\
\hline & & & & High-risk exploration test & Padilla et al. $(2016)^{29}$ \\
\hline & Non-human primate & Computerized monitoring & Behavior test & Go/NoGo visual discrimination test & Thorpe et al. $(1983)^{30}$ \\
\hline & & & & Fixed-ratio bar-press feeding task & Karádi et al. (1990) ${ }^{31}$ \\
\hline & & Behavioral monitoring & Behavior test & Taste reaction test & Rolls et al. $(1989)^{32}$ \\
\hline & Human & Computerized monitoring & Task & Stop signal reaction time task & Meule et al. $(2019)^{33}$ \\
\hline & & & & Go/NoGo task & Batterink et al. $(2010)^{34}$ \\
\hline & & & Survey & VARSEEK scale & Lähteenmäki and van Trijp (1995) \\
\hline & & Behavioral monitoring & - & - & - \\
\hline
\end{tabular}

VARSEEK, variety seeking tendency with respect to foods.

\section{Neuronal monitoring}

Several studies have been recently published regarding the preyrelated hunting behaviors. ${ }^{25,36}$ During hunting, superior colliculus to the zona incerta (ZI) neurons temporally correlates with predatory attacks but not the prey consumption phase. Activation of ZI GABAergic neurons strongly increase hunting of both live and artificial prey. ${ }^{25}$ However, these behaviors are more of predatory chasing behavior rather than food seeking one.

\section{Behavioral monitoring \\ High-risk exploration test}

Foot shock associated side of chamber presented food to mice during habituation and training. Even though mice could not access the food on the test day, they spent more time in the shock conditioned side. ${ }^{29}$ Although behavior tests to assess food seeking have been developed by multiple research groups, there are no well-defined and described methods for food seeking behavior. Some measured the time spent in food zone and latency to reach food zone. ${ }^{37,38}$ Other groups designated food-seeking as a lever pressing in operant conditioning. Recently, an assay to assess the ability to smell odorants has been developed, named as the buried food-seeking test which checks whether the mice could find the specific food hidden under the home cage bedding. There were tests that assessed foraging behavior in mice, although it was not directly related to food-seeking. Cricket hunting task was used to as- sess the latency of mouse to attack a cricket. Computer-controlled food-chasing task was a motor-driven task that involves a magnet glued to the bottom of the dish to assess initiation, chase, retrieval, consumption stage of mice. However, exact action of searching food has not been well assessed, which provides an opportunity for future researchers to develop.

\section{Non-human primate}

\section{Computerized monitoring}

\section{Go/NoGo visual discrimination test}

A study has outlined a Go/NoGo visual discrimination test, where a computer-controlled shutter would open to reveal the conditioned stimuli, which indicates that licking the tube would result either in aversive hypertonic solution or rewarding fruit juice. The reversal of the test, where the stimuli and the outcomes were reversed, was also performed. This task was selected for being known to be affected by lesions in orbitofrontal cortex. Neuron activities were studied during the task to see the involvement of orbitofrontal cortex in food seeking behaviors. ${ }^{30}$

\section{Fixed-ratio bar-press feeding task}

Frequently used operant task is a fixed-ratio bar-press feeding task. In some studies, the bar press task was divided into four stages: (1) the cue light stage where a red cue light indicates the beginning of the task, (2) the bar press stage where the subject has to 
press the bar by a fixed number, usually $20-30$ presses per reward, (3) the cue tone stage where a cue tone signifies the end of the task, and (4) the reward stage where food reward is given through food box. ${ }^{31}$ The firing rate of neurons were monitored during the task, and the behavioral response to stimuli were also recorded on paper chart. The bar press latency was observed and regarded to indicate the activeness of food seeking. ${ }^{20}$

\section{Behavioral monitoring}

Besides the willingness to perform an operant task, the taste reactions of subjects upon specific taste stimuli can be observed. In a study, a cynomolgous monkey resting in monkey chair received five taste stimuli applied to the tongue. Of the five stimuli, four are prototypes of four basic tastes, provoked by glucose, $\mathrm{NaCl}, \mathrm{HCl}$, and quinine hydrochloride. The fifth taste stimulus is blackcurrant juice, chosen because of its palatability and complexity of taste, which enables it to activate many gustatory neurons. The reaction of the monkey was classified into five categories, ranging from maximum rejection, clear rejection, neutrality, clear acceptance to maximum acceptance. Behaviors such as acid licking, swallowing, pursing of lips, tongue movement to eject delivered solution were observed to assess the willingness of subject to seek food. ${ }^{32}$

\section{Human}

\section{Computerized monitoring}

\section{Stop signal reaction time task}

It measures inhibition of a response that has already been initiated, that is, the ability to stop. It provides an estimate of the time taken to stop a response, the stop signal reaction time task, from measurable task parameters, the go-trial reaction-time distribution, and the accuracy of stopping on stop trials. ${ }^{33}$

\section{Go-NoGo task}

It investigates inhibitory control ability and impulsivity. Subjects respond (i.e., pressing a key) when go cue is presented and withhold a response (not pressing a key) in No-Go cue. Reaction time and error are measured and calculated to impulsivity and inhibitory control ability. ${ }^{34}$

\section{VARSEEK (variety seeking tendency with respect to foods) scale}

VARSEEK scale precisely assess the variety seeking bias of consumers. It is specifically designed for the food consumption context implying that it is not a personality characteristic that is generalizable across all product categories. The VARSEEK scale is based on the construct defined as the motivational factor targeting to provide variation in stimulant through diverse food product consumption, yet the instrumental or functional value of the food product alternatives. ${ }^{35}$

\section{Behavioral monitoring}

There is no behavioral experiment with noncomputerized task to measure only food seeking behavior of humans. Therefore, an appropriate behavioral experiment with noncomputerized task to assess food seeking behavior for humans needs to be developed.

\section{METHODS FOR EVALUATION OF FOOD CONSUMPTION BEHAVIOR}

\section{Rodent}

\section{Neuronal monitoring}

Neuronal activity during food consumption behavior can be monitored accurately by in vivo calcium imaging in mice. Photometry can measure bulk changes of calcium transients of genetically defined neurons..$^{39}$ Furthermore, miniature microscope would help distinguish aspects of appetitive or consummatory behaviors in the same neuronal population (Table 3). ${ }^{7}$

\section{Behavioral monitoring}

Consummatory behavior of mice usually is measured by the amount of food (or liquids) that has been consumed during certain amount of time (seconds, minutes, and hours). The difference in the weight of food (or food bowel) before and after the consummatory behavior is most frequently used method to measure the quantity of food consummatory behavior. Lickometer, a specialized device to measure the timing and quantity of licking, is another frequently used methods to measure the quantity of food consummatory behavior. 
Table 3. Methods for evaluation of food consumption behavior

\begin{tabular}{|c|c|c|c|c|c|}
\hline \multirow{2}{*}{$\begin{array}{l}\text { Feeding phase } \\
\text { Consumption }\end{array}$} & \multirow{2}{*}{$\begin{array}{r}\text { Species } \\
\text { Rodent }\end{array}$} & \multicolumn{3}{|c|}{ Monitoring method } & \multirow{2}{*}{$\begin{array}{c}\text { Author (year) } \\
\text { Cassidy et al. }(2019)^{40}\end{array}$} \\
\hline & & Neuronal monitoring & Tool & Optogenetics & \\
\hline & & & & Fiber photometry & Cassidy et al. (2019)40 \\
\hline & & & & Miniscope & Jennings et al. $(2015)^{7}$ \\
\hline & & Behavioral monitoring & Behavior test & Daily food intake & Dietrich et al. $(2015)^{9}$ \\
\hline & Non-human primate & Computerized monitoring & Behavior test & Automated feeder & Michopoulos et al. $(2016)^{41}$ \\
\hline & & Behavioral monitoring & Behavior test & Daily food intake & Ramsey et al. $(2005)^{42}$ \\
\hline & Human & Computerized monitoring & - & - & - \\
\hline & & Behavioral monitoring & Behavior test & Buffet test meal & Redman et al. $(2008)^{43}$ \\
\hline & & & & Microelectromechanical gyroscope & Dong et al. $(2012)^{44}$ \\
\hline & & & & Bogus taste test & Nijs et al. $(2010)^{45}$ \\
\hline
\end{tabular}

\section{Non-human primate \\ Computerized monitoring}

In several studies, the food intake of monkeys was monitored by automated feeder that displaces food pellets when active ted by radiofrequency chip implant. ${ }^{41,46,47}$ Each monkey has a subcutaneous implant of radiofrequency chip, which is read by the automated feeder which consequently displaces food pellet. The ship stores a nine-digit number, allowing the food intake of each monkey to be recorded. Additional monitoring such as video recording is involved to ensure that the monkey whose chip is read is actually the monkey who eats the food pellet.

\section{Behavioral monitoring}

Consummatory behavior in monkey is usually studied by monitoring the mass of the given food taken. The distinction between low and high fat diet, low and high calorie diet, etc. could be applied in the study design to study the effect of factors such as social stress and satiety on food intake.

\section{Human}

\section{Computerized monitoring}

There are no specific computerized tasks for human to assess food consumption behaviors.

\section{Behavioral monitoring}

The behavioral experiments with noncomputerized tasks for food consumption in human can be classified into two main categories; direct measurement and indirect measurement of food consumption.

\section{Direct measurement: Buffet test meal}

Buffet test meal is also called laboratory test-meal. This offers a pragmatic and impartial measure of calorie intake that may better be related to frequent eating pattern. ${ }^{48}$ This study applied 1-hour dinner sessions that were set between 4:00 PM and 6:00 PM. The test meals were composed of high- or low-fat forms of the same food categories as well as palatable buffet meal such as sweets, sweet-fats, and savory-fats. The participants were asked to be fasted at least 2 hours before attending the test-meal sessions. The total amount of consumption for each food was measured from pre- to post-meal to determine total meal intake. Also, the nutrient values were calculated based on the nutrition facts label of each food.

\section{Indirect measurement}

This consists of camera-enabled cell phones with data transfer capability. Participants were instructed to take photos of their food choice and leftovers with the labels on the pictures with their cell phones. Then, they were asked to send those photos to the researchers. There were four to six automated alarms for participants giving prompts to take photographs and to send them to the researchers.

\section{Using digital devices}

Microelectromechanical gyroscope (MEMS) tracks the number of bites, type of meals by detecting the wrist roll motion. The study using MEMS investigated three different conditions: (1) fixedsized pieces and menu (waffles), (2) relaxed condition (preferred food), and (3) outside of the lab (unrestricted settings; home, of- 
fice, restaurant, etc.). Although this method has some limitations since it is a preliminary study, this research has discovered that a simple pattern of wrist roll occurs during any bite. ${ }^{44}$ In addition, using smartphone applications (apps) to calculate the food consumption is the most accustomed method today. It is conducive for people to use the apps for daily food and exercise loggings. Logging the food diary is one of the behavior change techniques which infers that using smartphone apps can also be applied to lifestyle interventions. ${ }^{49}$

\section{Bogus taste test}

Participants were defined to five equal preweighed bowls containing high-calorie snacks. The chocolate, cookies, and cake were cut into small pieces to ease consumption. The bowls were displayed in random order. There was a questionnaire placed before each bowl asking about the taste of the food. The participants were given 15 minutes to taste attentively, one by one, and to assess the taste of each food on the questionnaires. They were directed notably that they could take food as much as they liked. They were not aware of the fact that their food (in kcal) was calculated by weighing afterwards. ${ }^{45}$

\section{CONCLUSION}

Obesogenic eating behavior is the major drive underlying epidemic surge of obesity in modern society. The decision to eat and the motivation to obtain food accompany multiple complicated food behaviors. Numerous methodologies have been developed to investigate multi-stages of eating behavior (craving, seeking and consumption) for rodents, non-human primates and humans.

Using these tools, basic researchers and clinical researchers would be able to dissect the mystery of eating behavior and obesity. These efforts will enlighten a more comprehensive clue to reveal the major pathogenic drive for obesity and find fundamental therapeutic drugs, devices and lifestyle modification protocols that will treat and prevent obesity.

\section{CONFLICTS OF INTEREST}

The authors declare no conflict of interest.

\section{ACKNOWLEDGMENTS}

This work was supported by the National Research Foundation of Korea (NRF) grant funded by the Korean Government (MIST; No. NRF-2018R1A5A2025964).

This work was supported by Creative-Pioneering Researchers Program through Seoul National University.

\section{AUTHOR CONTRIBUTIONS}

Study concept and design: YHL; drafting of the manuscript: all authors; critical revision of the manuscript: YHL; obtained funding: HJC; and study supervision: $\mathrm{HJC}$.

\section{REFERENCES}

1. Gropp E, Shanabrough M, Borok E, Xu AW, Janoschek R, Buch $\mathrm{T}$, et al. Agouti-related peptide-expressing neurons are mandatory for feeding. Nat Neurosci 2005;8:1289-91.

2. Chen Y, Lin YC, Kuo TW, Knight ZA. Sensory detection of food rapidly modulates arcuate feeding circuits. Cell 2015;160: 829-41.

3. Betley JN, Xu S, Cao ZF, Gong R, Magnus CJ, Yu Y, et al. Neurons for hunger and thirst transmit a negative-valence teaching signal. Nature 2015;521:180-5.

4. Harb MR, Almeida OF. Altered motivation masks appetitive learning potential of obese mice. Front Behav Neurosci 2014; 8:377.

5. Mandelblat-Cerf Y, Ramesh RN, Burgess CR, Patella P, Yang Z, Lowell BB, et al. Arcuate hypothalamic AgRP and putative POMC neurons show opposite changes in spiking across multiple timescales. Elife 2015;4:e07122.

6. Dayan P, Balleine BW. Reward, motivation, and reinforcement learning. Neuron 2002;36:285-98.

7. Jennings JH, Ung RL, Resendez SL, Stamatakis AM, Taylor JG, Huang J, et al. Visualizing hypothalamic network dynamics for appetitive and consummatory behaviors. Cell 2015;160:516-27.

8. Tzschentke TM. Measuring reward with the conditioned place preference (CPP) paradigm: update of the last decade. Addict Biol 2007;12:227-462. 
9. Dietrich MO, Zimmer MR, Bober J, Horvath TL. Hypothalamic Agrp neurons drive stereotypic behaviors beyond feeding. Cell 2015;160:1222-32.

10. Alhadeff AL, Su Z, Hernandez E, Klima ML, Phillips SZ, Holland RA, et al. A neural circuit for the suppression of pain by a competing need state. Cell 2018;173:140-52.

11. Fukuda M, Ono T. Amygdala-hypothalamic control of feeding behavior in monkey: single cell responses before and after reversible blockade of temporal cortex or amygdala projections. Behav Brain Res 1993;55:233-41.

12. Watanabe M, Cromwell HC, Tremblay L, Hollerman JR, Hikosaka K, Schultz W. Behavioral reactions reflecting differential reward expectations in monkeys. Exp Brain Res 2001;140: 511-8.

13. He Q, Xiao L, Xue G, Wong S, Ames SL, Schembre SM, et al. Poor ability to resist tempting calorie rich food is linked to altered balance between neural systems involved in urge and self-control. Nutr J 2014;13:92.

14. Johansson L, Ghaderi A, Andersson G. The role of sensitivity to external food cues in attentional allocation to food words on dot probe and Stroop tasks. Eat Behav 2004;5:261-71.

15. Brockmeyer T, Friederich HC, Küppers C, Chowdhury S, Harms L, Simmonds J, et al. Approach bias modification training in bulimia nervosa and binge-eating disorder: a pilot randomized controlled trial. Int J Eat Disord 2019;52:520-9.

16. Brockmeyer T, Hahn C, Reetz C, Schmidt U, Friederich HC. Approach bias and cue reactivity towards food in people with high versus low levels of food craving. Appetite 2015;95:197202.

17. Nijs IM, Franken IH, Muris P. The modified Trait and State Food-Cravings Questionnaires: development and validation of a general index of food craving. Appetite 2007;49:38-46.

18. Sobik L, Hutchison K, Craighead L. Cue-elicited craving for food: a fresh approach to the study of binge eating. Appetite 2005;44:253-61.

19. Kent KC. 'Medical cost savings through stroke prevention from 100 consecutive new carotid duplex scans', by G.S. Lavenson and D. Sharma, Cardiovascular Surgery, 1996, 4: 753-58. Cardiovasc Surg 1996;4:689-90.

20. Aou S, Takaki A, Karádi Z, Hori T, Nishino H, Oomura Y.
Functional heterogeneity of the monkey lateral hypothalamus in the control of feeding. Brain Res Bull 1991;27:451-5.

21. Stice E, Spoor S, Bohon C, Veldhuizen MG, Small DM. Relation of reward from food intake and anticipated food intake to obesity: a functional magnetic resonance imaging study. J Abnorm Psychol 2008;117:924-35.

22. Liu Y, Roefs A, Werthmann J, Nederkoorn C. Dynamics of attentional bias for food in adults, children, and restrained eaters. Appetite 2019; 135:86-92.

23. Cepeda-Benito A, Gleaves DH, Fernández MC, Vila J, Williams TL, Reynoso J. The development and validation of Spanish versions of the State and Trait Food Cravings Questionnaires. Behav Res Ther 2000;38:1125-38.

24. Chen Y, Knight ZA. Making sense of the sensory regulation of hunger neurons. Bioessays 2016;38:316-24.

25. Shang C, Liu A, Li D, Xie Z, Chen Z, Huang M, et al. A subcortical excitatory circuit for sensory-triggered predatory hunting in mice. Nat Neurosci 2019;22:909-20.

26.Zhao ZD, Chen Z, Xiang X, Hu M, Xie H, Jia X, et al. Zona incerta GABAergic neurons integrate prey-related sensory signals and induce an appetitive drive to promote hunting. Nat Neurosci 2019;22:921-32.

27. Chen Y, Essner RA, Kosar S, Miller OH, Lin YC, Mesgarzadeh $S$, et al. Sustained NPY signaling enables AgRP neurons to drive feeding. Elife 2019;8:e46348.

28. Machado CF, Reis-Silva TM, Lyra CS, Felicio LF, Malnic B. Buried food-seeking test for the assessment of olfactory detection in mice. Bio Protoc 2018;8:e2897.

29. Padilla SL, Qiu J, Soden ME, Sanz E, Nestor CC, Barker FD, et al. Agouti-related peptide neural circuits mediate adaptive behaviors in the starved state. Nat Neurosci 2016;19:734-41.

30. Thorpe SJ, Rolls ET, Maddison S. The orbitofrontal cortex: neuronal activity in the behaving monkey. Exp Brain Res 1983; 49:93-115.

31. Karádi Z, Oomura Y, Nishino H, Scott TR, Lénárd L, Aou S. Complex attributes of lateral hypothalamic neurons in the regulation of feeding of alert rhesus monkeys. Brain Res Bull 1990; 25:933-9.

32. Rolls ET, Sienkiewicz ZJ, Yaxley S. Hunger modulates the responses to gustatory stimuli of single neurons in the caudola- 
teral orbitofrontal cortex of the macaque monkey. Eur J Neurosci 1989; 1:53-60.

33. Meule A, Lender A, Richard A, Dinic R, Blechert J. Approachavoidance tendencies towards food: measurement on a touchscreen and the role of attention and food craving. Appetite 2019;137:145-51.

34. Batterink L, Yokum S, Stice E. Body mass correlates inversely with inhibitory control in response to food among adolescent girls: an fMRI study. Neuroimage 2010;52:1696-703.

35. Lähteenmäki L, van Trijp HC. Hedonic responses, variety-seeking tendency and expressed variety in sandwich choices. Appetite $1995 ; 24: 139-51$.

36. Li Y, Zeng J, Zhang J, Yue C, Zhong W, Liu Z, et al. Hypothalamic circuits for predation and evasion. Neuron 2018;97:91124.

37. Burnett CJ, Li C, Webber E, Tsaousidou E, Xue SY, Brüning JC, et al. Hunger-driven motivational state competition. Neuron 2016;92:187-201.

38. Zhang X, van den Pol AN. Rapid binge-like eating and body weight gain driven by zona incerta GABA neuron activation. Science 2017;356:853-9.

39. Resendez SL, Stuber GD. In vivo calcium imaging to illuminate neurocircuit activity dynamics underlying naturalistic behavior. Neuropsychopharmacology 2015;40:238-9.

40. Cassidy RM, Lu Y, Jere M, Tian JB, Xu Y, Mangieri LR, et al. A lateral hypothalamus to basal forebrain neurocircuit promotes feeding by suppressing responses to anxiogenic environmental cues. Sci Adv 2019;5:eaav1640.

41. Michopoulos V, Diaz MP, Wilson ME. Social change and access to a palatable diet produces differences in reward neurochemistry and appetite in female monkeys. Physiol Behav 2016; 162:102-11.
42. Ramsey JJ, Kemnitz JW, Newton W, Hagopian K, Patterson TA, Swick AG. Food intake in rhesus monkeys following central administration of orexins. Regul Pept 2005;124:209-14.

43. Redman LM, Martin CK, Williamson DA, Ravussin E. Effect of caloric restriction in non-obese humans on physiological, psychological and behavioral outcomes. Physiol Behav 2008; 94:643-8.

44. Dong Y, Hoover A, Scisco J, Muth E. A new method for measuring meal intake in humans via automated wrist motion tracking. Appl Psychophysiol Biofeedback 2012;37:205-15.

45. Nijs IM, Muris P, Euser AS, Franken IH. Differences in attention to food and food intake between overweight/obese and normal-weight females under conditions of hunger and satiety. Appetite 2010;54:243-54.

46. Godfrey JR, Diaz MP, Pincus M, Kovacs-Balint Z, Feczko E, Earl E, et al. Diet matters: glucocorticoid-related neuroadaptations associated with calorie intake in female rhesus monkeys. Psychoneuroendocrinology 2018;91:169-78.

47. Wilson ME, Fisher J, Fischer A, Lee V, Harris RB, Bartness TJ. Quantifying food intake in socially housed monkeys: social status effects on caloric consumption. Physiol Behav 2008;94: 586-94.

48. Fearnbach SN, Thivel D, Meyermann K, Keller KL. Intake at a single, palatable buffet test meal is associated with total body fat and regional fat distribution in children. Appetite 2015;92: 233-9.

49. Toro-Ramos T, Lee DH, Kim Y, Michaelides A, Oh TJ, Kim $\mathrm{KM}$, et al. Effectiveness of a smartphone application for the management of metabolic syndrome components focusing on weight loss: a preliminary study. Metab Syndr Relat Disord 2017;15:465-73. 